\title{
Evidence for a finite compressibility of a quasi-one-dimensional ballistic channel
}

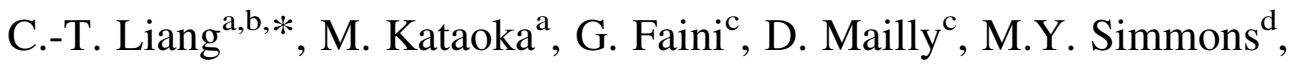 \\ A.W. Rushforth ${ }^{\mathrm{a}}$, C.G. Smith ${ }^{\mathrm{a}}$, D.A. Ritchie ${ }^{\mathrm{a}}$, M. Pepper ${ }^{\mathrm{a}}$ \\ ${ }^{a}$ Cavendish Laboratory, Madingley Road, Cambridge CB3 OHE, UK \\ ${ }^{\mathrm{b}}$ Department of Physics, National Taiwan University, Taipei 106, Taiwan, ROC \\ ${ }^{c}$ L2M-CNRS, 196 Avenue.H. Ravera, 92220 Bagneux, France \\ ${ }^{\mathrm{d}}$ School of Physics, University of New South Wales, Sydney 2052, NSW, Australia
}

Available online 19 April 2005

\begin{abstract}
It is known that the local conditions in an electrical circuit can affect a nearby, but electrically isolated circuit. Such a 'noninvasive' measurement technique can be employed to study the screening ability (compressibility) of an electron system. In this work, we use a quasione-dimensional ballistic channel to screen an electric field between a gate and lateral quantum dot. Our results clearly show that the screening ability decreases with decreasing number of occupied 1D channel. When there are a large number of 1D subbands occupied in the channel, the screening ability tends to saturate, approaching its 2D limit.
\end{abstract}

(C) 2005 Elsevier Ltd. All rights reserved.

\section{Introduction}

By using the electrostatic squeezing technique [1], it is possible to define a lateral quantum dot which confines electrons in an isolated region within a two-dimensional electron gas (2DEG). Consider a lateral quantum dot [2] weakly coupled to the source and drain contacts where the tunnelling conductance through the $\operatorname{dot} G$ is low, $G \ll 2 e^{2} / h$. If the energy required for adding an extra electron to the quantum dot is much higher than the chemical potentials in the source and drain contacts, transport through the quantum dot is prohibited. This is the Coulomb blockade of single electron tunneling [3]. By changing the applied gate voltage to align the energy state required for adding an extra electron in the dot, with the chemical potentials in the leads, transport through the dot can occur via single electron

\footnotetext{
* Corresponding author. Address: Cavendish Laboratory, Madingley Road, Cambridge CB3 OHE, UK.

E-mail address: ctliang@phys.ntu.edu.tw (C.-T. Liang).
}

0026-2692/\$ - see front matter (C) 2005 Elsevier Ltd. All rights reserved. doi:10.1016/j.mejo.2005.02.110 tunnelling. Therefore one observes a series of Coulomb oscillations periodic in gate voltage [3].

It is well established that a grounded metal plate has a perfect screening ability from an external electric field. However, the situation is different when considering a 2DEG formed at the interface of a GaAs/AlGaAs heterostructure. It has been shown that a GaAs 2DEG can only partially screen an external electric field from an electrode [4]. In the seminal work of Luryi [5], it has been suggested that partial penetration of an electric field through a highly conductive 2DEG allows the implementation of novel highspeed devices, such as a three-terminal resonant tunnelling transistor and a gated-controlled thermionic emission transistor.

It is known that the local conditions in an electrical circuit can affect a nearby, but electrically isolated circuit [6]. One such class of 'noninvasive' measurement is the determination of the screening ability of an electron system as mentioned earlier. Using both screening in GaAs 2DEGs, and Coulomb oscillations observed in a quantum dot in a double 2DEG, Field and co-workers have studied a quantum dot shaped in the upper 2DEG in which Coulome oscillations in the quantum dot is observed as a function 
of the applied back-gate voltage [7]. The period of the observed Coulomb oscillations varies as the penetrating electric field through the lower 2DEG is changed. In this way, Coulomb oscillations can be served as a noninvasive probe of local density of states in the lower 2DEG.

In this paper, we study a quasi-one-dimensional ballistic channel in close proximity to a lateral quantum dot in a separate and electrically isolated circuit $[6,8]$. We use a 1D channel to screen an electric field between a gate and a lateral quantum dot. In our case, Coulomb blockade oscillations observed in our quantum dot can be used as a noninvasive probe of screening of the $1 \mathrm{D}$ channel. Our experimental results demonstrates that a quasi-one-dimensional channel has a finite screening ability which decreases with decreasing number of the occupied $1 \mathrm{D}$ subbands in the ballistic channel.

\section{Experimental}

The device that we studied comprises of a 1D channel in close proximity to a lateral quantum dot in an electrically isolated circuit, as shown in the inset to Fig. 1. There is an insulating layer between G3 and the two overlaying finger gates (G4 and G5) so all the gates can be independently controlled [9-11]. By applying sufficiently large negative biases on G2, G3, G4 and G5, a lateral quantum dot can be shaped in circuit on the left hand side. A quasi-ballistic ballistic constriction can be shaped by applying large enough negative biases on both G2 and G1. The carrier concentration of the 2DEG was $3.3 \times 10^{15} \mathrm{~m}^{-2}$ with a mobility of $750 \mathrm{~m}^{2} / \mathrm{Vs}$ after illumination. The depth of the 2DEG is $300 \mathrm{~nm}$. Experiments were performed in dilution refrigerator at a base temperature $T \sim 50 \mathrm{mK}$. Two-terminal

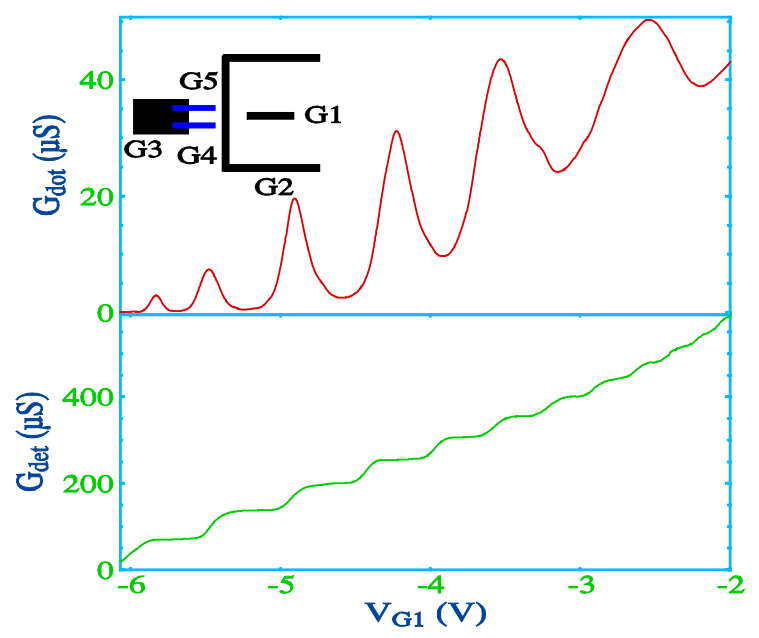

Fig. 1. Conductance $\left(G_{\mathrm{det}}\right)$ measurements of channel (upper panel) and conductance measurement of the quantum dot $\left(G_{\mathrm{dot}}\right.$ :lower panel) as a function of the voltage $V_{\mathrm{G} 1}$ applied on $(\mathrm{G} 1)$ at $V_{\mathrm{G} 3}=-4 \mathrm{~V}, V_{\mathrm{G} 4}=-8 \mathrm{~V}$, $V_{\mathrm{G} 5}=-9 \mathrm{~V}$ and $V_{\mathrm{G} 3}=-3 \mathrm{~V}$, respectively. The inset shows a schematic diagram of the device. conductances of both the channel and quantum dot were measured simultaneously using standard phase-sensitive ac lock-in techniques.

\section{Results and discussion}

Fig. 1 shows the conductances of the lateral quantum dot $\left(G_{\mathrm{dot}}\right)$ and the quasi-ballistic 1D channel $\left(G_{\mathrm{det}}\right)$ measured simultaneously as a function of the voltage applied on G1 $\left(V_{\mathrm{G} 1}\right)$. As shown in the lower panel, clean ballistic conductance steps are observed in the circuit on the right hand side. This result demonstrates that we have a clean quasi-one-dimensional in which impurity scattering is negligible [12-14]. We can see that the conductance through the lateral quantum dot shown in the upper channel show Coulomb oscillations [3]. The conductance maxima can be ascribed to single-electron tunnelling through our quantum dot whereas the conductance minima can be ascribed to the situation when the conduction through the quantum dot is suppressed. We note that in contrast to conventional Coulomb oscillations which are periodic in gate voltage, in our case, the separation between adjacent conductance maxima in gate voltage $V_{\mathrm{G} 1}$ decreases with decreasing $V_{\mathrm{G} 1}$. Our results can be explained by the finite screening ability of a 1D channel. If the 1D channel perfect screens the electric field emanating from G1, the conductance through the quantum dot would not be varied as a function of $V_{\mathrm{G} 1}$. In our case, the electric field from G1 penetrates the 1D channel as the 1D channel has a finite screening ability. When there are a large (small) number of 1D subbands occupied in the channel, screening is strong (weak) therefore in order observe a pair of conductance peaks, the required electric field and hence gate voltage is large (small).

To further investigate the screening effect of the quasione-dimensional channel, we plot the adjacent peak separation in $V_{\mathrm{G} 1}$ as a function of the number of occupied 1D subband in the channel. These results are obtained at

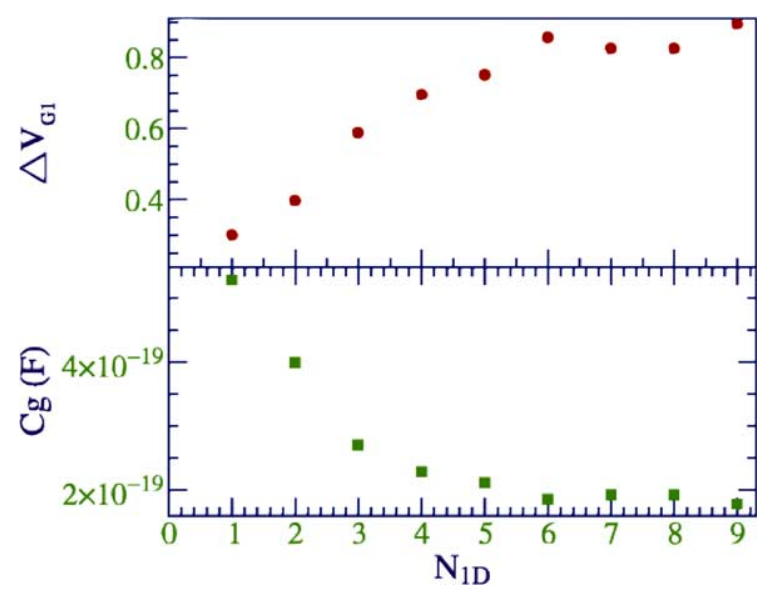

Fig. 2. Upper panel: the peak separation in $V_{\mathrm{G} 1}$ as a function of the number of 1D subbands occupied in the channel $N_{1 \mathrm{D}}$. Lower panel: the corresponding gate capacitance $C_{\mathrm{g}}$ as a function of $N_{\mathrm{lD}}$. 
different $V_{\mathrm{G} 3}$ so that two adjacent conductance peaks coincide with a conductance plateau observed in $G_{\text {det }}$. The gate-dot capacitance $C_{\mathrm{g}}$ reflects the screening ability of the 1D channel in the nearby, but electrically isolated circuit. The larger $C_{\mathrm{g}}$, the smaller screening ability. We can see that the peak separation tends to saturate at high $n$. This suggests that when there are a large number of occupied 1D subbands $\left(N_{1 \mathrm{D}} \geq 6\right)$, the screening ability is approximately constant and approaches the 2D limit [7] (Fig. 2).

\section{Conclusions}

In summary, we have presented experimental evidence for a finite screening ability of a quasi-1D ballistic channel using a non-invasive measurement technique. Our results clearly show that the screening ability decreases with decreasing number of occupied $1 \mathrm{D}$ channel. When there are a large number of 1D subbands occupied in the channel, the screening ability tends to saturate, approaching its 2D limit.

\section{Acknowledgements}

This work was funded by the EPSRC and the Royal Society, United Kingdom. C.T.L. acknowledges financial support from the NSC, Taiwan (42006F and NSC 92-2112M-002-029).

\section{References}

[1] T.J. Thornton, M. Pepper, H. Ahmed, D. Andrews, G.J. Davies, Phys. Rev. Lett. 56 (1986) 1198.

[2] C.G. Smith, M. Pepper, H. Ahmed, J.E.F. Frost, D.G. Hasko, D.C. Peacock, D.A. Ritchie, G.A.C. Jones, J. Phys. C 21 (1988) L893.

[3] U. Meirav, M.A. Kastner, S.J. Wind, Phys. Rev. Lett. 65 (1990) 771.

[4] J.P. Eisenstein, L.N. Pfeiffer, K.W. West, Phys. Rev. B 50 (1994) 1760.

[5] S. Luryi, Appl. Phys. Lett. 52 (1987) 501.

[6] M. Field, C.G. Smith, M. Pepper, D.A. Ritchie, J.E.F. Frost, G.A.C. Jones, D.G. Hasko, Phys. Rev. Lett. 70 (1993) 1311.

[7] M. Field, C.G. Smith, M. Pepper, K.M. Brown, E.H. Linfield, M.P. Grimshaw, D.A. Ritchie, G.A.C. Jones, Phys. Rev. Lett. 77 (1996) 350.

[8] R. Nemutudi, M. Kataoka, C.J.B. Ford, N.J. Appleyard, M. Pepper, D.A. Ritchie, G.A.C. Jones, J. Appl. Phys. 95 (2004) 2557.

[9] C.-T. Liang, M.Y. Simmons, C.G. Smith, D.A. Ritchie, M. Pepper, Phys. Rev. Lett. 81 (1998) 3507.

[10] C.-T. Liang, M.Y. Simmons, C.G. Smith, D.A. Ritchie, M. Pepper, Phys. Rev. B 60 (1999) 10687.

[11] C.-T. Liang, O.A. Tkachenko, V.A. Tkachenko, D.G. Baksheyev, M.Y. Simmons, D.A. Ritchie, M. Pepper, Phys. Rev. B 70 (2004) 195324.

[12] J.T. Nicholls, J.E.F. Frost, M. Pepper, D.A. Ritchie, M.P. Grimshaw, G.A.C. Jones, Phys. Rev. B 48 (1994) 8866.

[13] C.-T. Liang, I.M. Castleton, J.E.F. Frost, C.H.W. Barnes, C.G. Smith, C.J.B. Ford, D.A. Ritchie, M. Pepper, Phys. Rev. B 55 (1997) 6723.

[14] C.-T. Liang, M. Pepper, M.Y. Simmons, C.G. Smith, D.A. Ritchie, Phys. Rev. B 61 (2000) 9952. 